\title{
Effect of the Tensioactive Agent SDS on Extender's Boar Semen Viability after Thawing
}

\author{
Maria Helena Moreira da Silva, Loide Valadão and Fernando Moreira da Silva \\ Department of Animal Reproduction, Faculty of Agrarian Sciences and Environment Animal, \\ University of the Azores, CITA-A, 9701-851 Angra do Heroísmo, Portugal
}

\author{
Article history \\ Received: 30-11-2020 \\ Revised: 15-01-2021 \\ Accepted: 20-01-2021 \\ Corresponding Author: \\ Fernando Moreira da Silva \\ Department of Animal \\ Reproduction, Faculty of \\ Agrarian Sciences and \\ Environment Animal, \\ University of the Azores, \\ CITA-A, 9701-851 Angra do \\ Heroísmo, Portugal \\ Email: Joaquim.fm.silva@uac.pt
}

\section{Introduction}

A large part of the boar's population remains unqualified for semen freezing programs because of unsatisfactory post-thaw sperm quality and fertility rates. Therefore, the achievement of successful semen cryopreservation would represent a dramatic leap in swine production systems, as it would allow the widespread use of germplasm banks for storing semen doses from selected males, for preserving genetic diversity and for conserving rare breeds. Besides cryopreservation of spermatozoa has been a great resource for the trade-in genetic material and gene banks, cooling and freezing are traumatic events for spermatozoa, although the extent of these effects varies with the species. Freezing of boar spermatozoa started already by the $1960 \mathrm{~s}$, based on previous experience in dairy cattle (Polge et al., 1949), yielding, however, much lower reproductive performances than in bovine. From then, the increasing use of cryopreserved boar semen has motivated the research and development of extenders, cryoprotectants and freezing protocols, intending the preservation of semen in optimal conditions after thawing, but unfortunately results still not satisfactory and a breakthrough in a commercial application has not yet occurred. Moreover, while several additives and changes in the cooling curve and thawing have been tested, the overall procedure is still the same as purposed by Eriksson and Rodríguez-Martínez twenty years ago (Eriksson and Rodriguez-Martinez, 2000). Although its use in some commercial production applications such as genetic transfer projects, frozen boar semen has not been used under production conditions as efficiently as liquidpreserved semen, due to the high susceptibility of boar spermatozoa to damage during cryopreservation and a complicated process of deep freezing. Membranes are thought to be the main problem of chilling or freezing damage in cells (Morris and Clarke, 1981). The thermal stress placed on membranes during cooling has been hypothesized to result in transitions from the liquid crystalline to the gel phase, which has substantial deleterious consequences. As a result of this transition, the cell may leak its contents to the surrounding medium 
(Crowe et al., 1988), possibly due to defects in the bilayer between the gel and liquid crystalline domains.

In recent years, the development of freezing techniques for semen cryopreservation includes various strategies for improvement of the quality of frozen/thawed spermatozoa and to increase the number of spermatozoa available for fertilization. Therefore, a few studies evaluate the addition of surfactants as they act as synthetic detergents and, a wetting agent, which is integrated into the freezing medium improves the efficiency of lipid-based extenders, enhancing cryosurvival of boar spermatozoa after thawing (Vilagran Martí, 2016). This combination, surfactant and egg yolk, provides a better protection effect, as the detergent modifies egg yolk particles, enhancing their interaction with sperm plasma membrane (Holt, 2000; Rodriguez-Martinez and Wallgren, 2011).

Tensioactive agents or surfactants are specific chemicals characterized by their structural bipolarity: One hydrophobic group and another hydrophilic group. They are classified as anionic, cationic or, non-ionic surfactants and have various uses in healthcare, such as excipients to dissolve hydrophobic drugs in aqueous solutions or to compound injectable emulsions. Their interest in chemical decontamination is closely related to their ability to dissolve both hydrophilic and hydrophobic drugs and then to be wiped on tissues. Sodium Dodecyl Sulfate (SDS) is a surfactant that in the diluent has proved beneficial for the cryopreservation of sperm from several domestic and wildlife species (Holt, 2000). Addition of SDS to the freezing medium protected boar sperm cells against freezing damage and led to vastly improved post-thaw sperm quality. Although fertility is not highly related to fertility outcomes such as farrowing rate and litter size, the use of poor-quality semen is related to reduced fertility.

Therefore, the aim of the present study was to evaluate the viability of boar semen, after thawing, using the anionic surfactant SDS in the freezing medium.

\section{Materials and Methods}

Once in the lab, semen samples were processed, essentially using the straw-freezing procedure described by Westendorf and collaborators (Westendorf et al., 1975) with minor modifications. Briefly, semen was placed at $15^{\circ} \mathrm{C}$ for $2 \mathrm{~h}$ and then centrifuged at $800 \times \mathrm{g}$ for $10 \mathrm{~min}$ also at $15^{\circ} \mathrm{C}$. After that, most of the supernatant was discarded leaving $3.5 \mathrm{~mL}$ of the supernatant in each flask to resuspend semen pellets to evaluate its concentration in a Neubauer chamber as afore-described. Then, $6 \times 500 \mu \mathrm{L}$ aliquots of the resuspended semen were placed in each of 6 falcon tubes and $1 \mathrm{~mL}$ of all the chemicals and media were purchased from Sigma Chemicals Co. (St Louis, MO) and disposable plastic wares were from Nunc (Roskilde, Denmark) unless otherwise mentioned.
Pigs used in the present experiment belonged to the Artificial Insemination Centre of the Autonomous Government of the Azores-Portugal, which were handled following international ethical rules. All animals were provided ad libitum access to water and fed with commercial diets, according to the nutritional requirements for adult boars. Boar's ejaculates were collected twice a week, using the glove-hand method through a layer of gauze into a thermos bottle to remove gel particle, maintained at $37^{\circ} \mathrm{C}$. From each boar and without any interference in the semen collection routine, barely $10 \mathrm{~mL}$ spermatozoa-rich fraction was used for freezing, while the rest of the ejaculated spermatozoa was used for routine production of liquid semen doses, thus combining production and banking. Immediately after collection, a macroscopic assessment was performed, evaluating the volume, appearance, color, $\mathrm{pH}$ and agglutination level. Then, a drop of semen was observed by contrast-phase microscopy at low magnification $(\times 10)$ to assess the massal motility graded from 0 to $5(0=$ no movement, 5 = Massal motility with vortex). Sperm concentration was evaluated in a Neubauer chamber, using a dilution of $10 \mu \mathrm{L}$ of semen to $990 \mu \mathrm{L}$ of water. The sperm vitality was assessed by counting after the one-step eosin-nigrosin (Fluka Chemie GmbH, Sigma-Aldrich, Switzerland), staining technique, to determine the percentage of live (eosin-negative) spermatozoa according to (Barth and Oko, 1989) and assessment of cellular elements other than spermatozoa. Sperm morphology was also performed, according to (Rusu et al., 2009) and spermatozoon was considered normal when its head, tail and all borderline forms were considered standard. Only semen classified with massal motility and viability higher than 4 and $60 \%$, respectively, were used in the experiment.

Gaczarzewicz et al. (2016), evaluation of agglutination indicated what percent of the sperm cells in the field of view under low magnification are stuck to each other. This evaluation was performed at the same time as motility/morphology evaluation, using the categories as described in Table 1. Ejaculates with a score 3 were also considered an elimination factor as this can indicate some problems in the fertility of the ejaculate.

After the evaluation afore-described, if the semen showed quality to be frozen, it was versed in flasks, placed in isolated containers at $37^{\circ} \mathrm{C}$ and transported to the lab being processed about $10 \mathrm{~min}$ after collection.

Table 1: System used to score of boar semen ejaculate agglutination (Gaczarzewicz et al., 2016)

\begin{tabular}{ll}
\hline Score & $\begin{array}{l}\text { Percentage of agglutinated } \\
\text { cells within a field of view }\end{array}$ \\
\hline 0 & None \\
1 & Mild $(<10 \%)$ \\
2 & Moderate $(10-25 \%)$ \\
3 & Severe $(>25 \%)$ \\
\hline
\end{tabular}


Once in the lab, semen samples were processed, essentially using the straw-freezing procedure described by Westendorf and collaborators 11 with minor modifications. Briefly, semen was placed at $15^{\circ} \mathrm{C}$ for $2 \mathrm{~h}$ and then centrifuged at $800 \times \mathrm{g}$ for $10 \mathrm{~min}$ also at $15^{\circ} \mathrm{C}$. After that, most of the supernatant was discarded leaving $3.5 \mathrm{~mL}$ of the supernatant in each flask to resuspend semen pellets to evaluate its concentration in a Neubauer chamber as afore-described. Then, $6 \times 500 \mu \mathrm{L}$ aliquots of the resuspended semen were placed in each of 6 falcon tubes and $1 \mathrm{~mL}$ of MRA extender, supplemented with $3 \%$ glycerol, $10 \%$ of egg yolk and different concentrations of SDS $(0.000 ; 0.025 ; 0.050 ; 0.10 ; 0.20$ and $0.40 \% \mathrm{w} / \mathrm{v})$ was respectively added to each tube and sperm viability was evaluated. Then straws were filled, incubated for $2 \mathrm{~h}$ at $5^{\circ} \mathrm{C}$ and frozen in a programmable freezer (Ice-Cube14S; SY-LAB,149 Neupurkersdorf, Austria) with the starting temperature of $5^{\circ} \mathrm{C}$, with a programmed curve, as follow: From 5 to $-5^{\circ} \mathrm{C}$ at $-6^{\circ} \mathrm{C} / \mathrm{min}$, from -5 to $-80^{\circ} \mathrm{C}$ at $-40^{\circ} \mathrm{C} / \mathrm{min}$ in which semen was for $30 \mathrm{~min}$. From -80 to $-150^{\circ} \mathrm{C}$ semen was cooled at a slope of $-60^{\circ} \mathrm{C} / \mathrm{min}$ being then plunged in liquid nitrogen.

Thawing was carried out seven days after freezing, always in the same way, by immersing the straws in a circulating water bath at $37^{\circ} \mathrm{C}$ for $30 \mathrm{sec}$ (Silva et al., 2015), versed in Eppendorf tubes and evaluated for the same parameters as afore-described.

\section{Results}

Results indicated that, after collecting, sperm obtained from the different boars were always alkaline and values very similar among boars. On average, $\mathrm{pH}$ was $7.50 \pm 0.27$ ranging from 7.05 to 7.79 . Viability, motility and agglutination level were respectively $62.7 \pm 9.9 \% ; 4.8 \% \pm 0.3$ and $9.3 \% \pm 1.82$. In the $4 \mathrm{~h}$ refrigeration process in which semen was diluted with the different extenders, no loss of viability, motility, or agglutination was observed, showing that, even for the highest concentrations of SDS used, no chemical toxicity occurred (Table 2). After thawing, it has been observed that SDS statistically increased $(\mathrm{P}<0.05)$ sperm viability (Fig. 1) as well as sperm motility (Fig. 2).

For semen viability, up to the concentration of 0.05 SDS (\%W/V), no statistical differences were observed among treatments. After that viability increased to $21.6 \% \pm 0.75$ and $30.1 \% \pm 2.15$ for the SDS concentrations of 0.1 and 0.2 , respectively. At the highest concentration of SDS used, a decrease in sperm viability was observed, dropping to values identical to the control $(16.6 \% \pm 1.0)$.

Table 2: Results of boar sperm viability as evaluated by the eosin/nigrosin test and motility graded from 0 to 5 ( $0=$ no movement, 5 = Massal motility with vortex) after collecting and before freezing. Data represent Mean \pm SEM of twelve boars, each one collected 3 times

\begin{tabular}{|c|c|c|c|c|}
\hline \multirow[b]{2}{*}{ Treatment } & \multicolumn{2}{|c|}{ After collecting } & \multicolumn{2}{|c|}{ Before freezing } \\
\hline & Viability & Motility & Viability & Motility \\
\hline Control & $62.71 \pm 4.02$ & $4.8 \pm 0.13$ & $62.0 \pm 2.21$ & $4.54 \pm 0.46$ \\
\hline $0.025 \% \mathrm{w} / \mathrm{v}$ & $62.71 \pm 4.02$ & $4.8 \pm 0.13$ & $61.01 \pm 3.04$ & $4.65 \pm 0.49$ \\
\hline $0.050 \% \mathrm{w} / \mathrm{v}$ & $62.71 \pm 4.02$ & $4.8 \pm 0.13$ & $61.40 \pm 2.05$ & $4.65 \pm 0.52$ \\
\hline $0.10 \% \mathrm{w} / \mathrm{v}$ & $62.71 \pm 4.02$ & $4.8 \pm 0.13$ & $62.30 \pm 4.09$ & $4.62 \pm 0.62$ \\
\hline $0.20 \% \mathrm{w} / \mathrm{v}$ & $62.71 \pm 4.02$ & $4.8 \pm 0.13$ & $60.08 \pm 5.02$ & $4.62 \pm 0.58$ \\
\hline $0.40 \% \mathrm{w} / \mathrm{v}$ & $62.71 \pm 4.02$ & $4.8 \pm 0.13$ & $61.97 \pm 3.70$ & $4.70 \pm 0.58$ \\
\hline
\end{tabular}

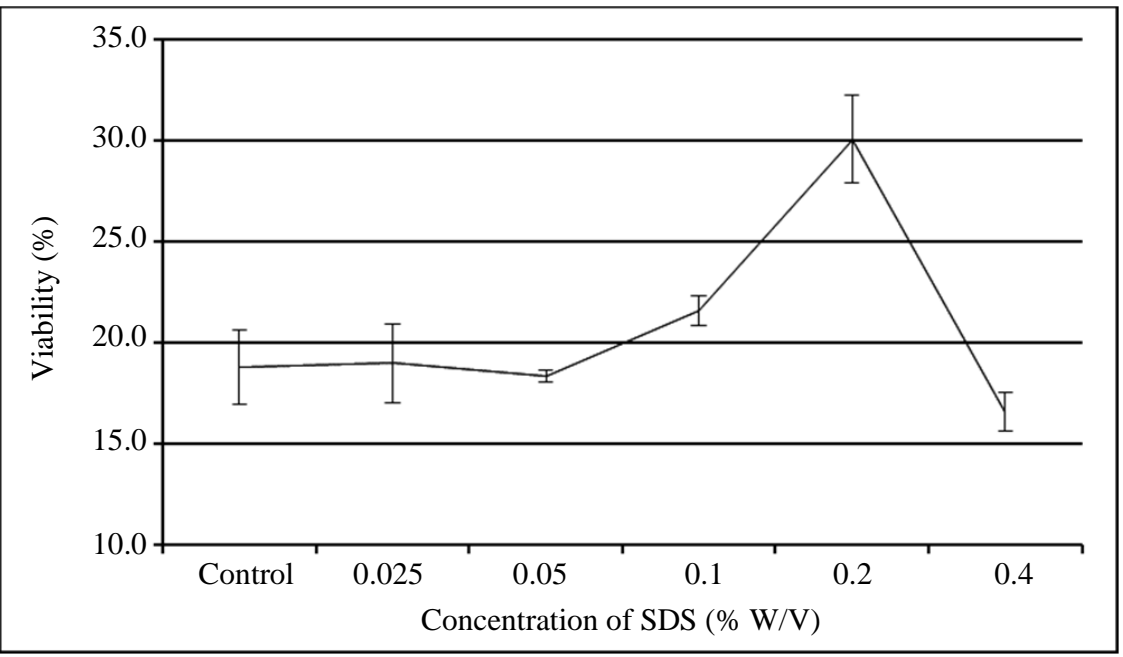

Fig. 1: Boar semen viability, thawed one week after freezing, as evaluated by the eosin/nigrosin test. Data represent Mean \pm SD of twelve boars, each one collected 3 times 


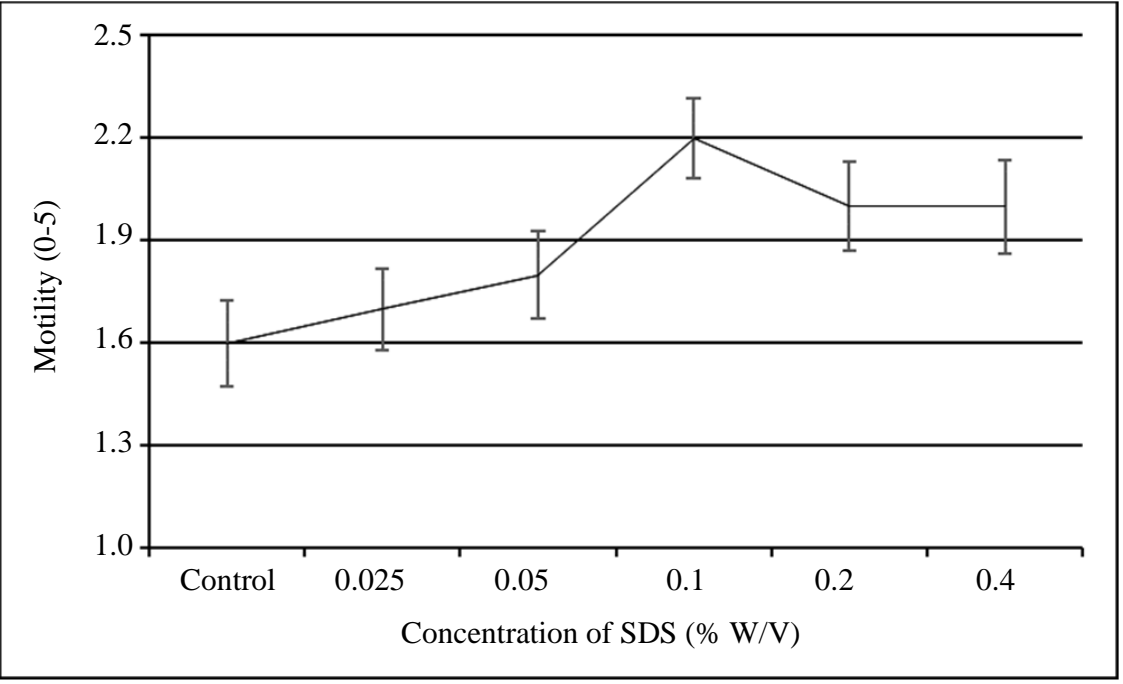

Fig. 2: Boar semen motility (0-5) thawed one week after freezing. Data represent Mean \pm SD of twelve boars, each one collected 3 times

As far as semen motility is concerned, the values followed the same trend observed for sperm viability. For the control and the lowest concentrations of SDS, there were no significant differences, after which a maximum motility value was reached for the 3 highest concentrations, being these values statistically different from the control $(\mathrm{P}<0.05)$.

\section{Discussion}

Cryopreservation of boar semen is not common, as the damage caused to the cells is extensive. The experiments reported here aimed to consider whether SDS would affect boar sperm viability and motility after freezing/thawing. Sperm of 12 adult boars were collected, three times each, equilibrated for $2 \mathrm{~h}$ and incubated for $2 \mathrm{~h}$ with egg yolk and different concentrations of SDS from 0 to $0.4 \% \mathrm{~W} / \mathrm{V}$ and then frozen in a programmable freezer. Results indicated that SDS improved motility and viability of sperm at concentrations of 0.1 and 0.2 where the viability/motility was statistically superior to the control. As known, egg yolk and SDS are non-permeable cryoprotectants used in freezing extender providing a protective effect to spermatozoa and improving post thawed sperm quality (Pursel et al., 1978). Besides egg yolk is a common ingredient of mammalian semen extender to protect sperm against initial cold shock, (Buhr and Pettitt, 1995) suggested that SDS enhances the cryoprotective properties of the egg yolk to protect the sperm membrane from cryoinjuries, because the addition of SDS in the extender could mix and increase molecules dispersion from the yolk. This could result in an optimum contact between phospholipid of yolk and plasma membrane of spermatozoa, increasing the semen quality.
The first attempt in which SDS was added as a surfactant to improve semen viability after freezing, was developed by Paquin and collaborators (Paquin et al., 1984), working on Human semen. In their research, it has been proved that beyond a neutral-glucosidase isoenzyme, which originates in the epididymis, seminal plasma contains an acid isoenzyme contributed by the prostate (Cooper et al., 1990) which can be selectively inhibited by the SDS. The specific role of $\alpha$-glucosidase in sperm function is still unknown. However, it has been suggested that it could modify sperm surface glycoproteins during epididymal transit, including a glycoprotein that is possibly involved in sperm-egg binding (Ali et al., 1994). The enzyme has been localized in the cytoplasm of the efferent duct epithelium and the brush border of the human epididymis in its entire length (Yeung et al., 1990). Moreover, as known, lipids from whole sperm and sperm plasma membrane may partially affect the cryogenic survival of sperm (De Leeuw et al., 1991). Differences in fatty acid composition have been associated with tolerance to coldshock and cryopreservation. Specifically, a higher ratio of unsaturated/saturated membrane fatty acids and lower levels of cholesterol within bull and ram sperm membranes as compared with sperm membranes from humans and dogs have been suggested as an explanation for the differences in cold shock and cryopreservation tolerance observed between these species (White, 1993). However, these species-to-species differences in membrane lipid composition can neither explain the major differences in post-thaw survival and fertility between species such as bull and boar, nor the variation between semen quality and the ability to freeze between breeds as well as between individual boars and even the season. In our experiment, however, no great differences 
have been observed among boars, although approximately 40 to $50 \%$ of boar spermatozoa do not survive cryopreservation. Some research indicated that the loss of viability after thawing can be the fact that boar spermatozoa are very susceptible to peroxidative damage because of the high content of polyunsaturated fatty acids (Esmaeili et al., 2015) and low level of cholesterol in the plasma membrane (Cerolini et al., 2000).

During slow freezing, boar spermatozoa are also sensitive to cellular injury and mechanical stress caused by ice formation around the cells (Rodriguez-Martinez and Wallgren, 2011; Di Santo et al., 2011). It results in membrane reorganizations that can disrupt membrane integrity, structure and function, contributing to cell death after thawing. On the other hand, the variability of semen to sustain sperm cryopreservation could be related to differences in the seminal plasma composition (Roca et al., 2006). As in our experiment, only a small fraction of semen was left after centrifugation, which may justify the similarity obtained between the different boars used in the study. Moreover, considering that the boars were all the same breed, kept under identical management conditions and collected in the same season, these facts contribute to the semen certainly maintaining the same physical and chemical characteristics.

Identical experiments as presented in our study have been developed for horse semen by El-Badry and collaborators assuming that the addition of appropriate concentration of SDS is fundamental for equine semen cryopreservation. SDS, egg yolk and glycerol at concentrations of $0.03,15$ and $5 \%$, respectively, reduced the cryodamage of stallion sperm (El-Badry et al., 2014). The precise mechanisms by which SDS improves sperm cryosurvival remain unknown. However, it has been demonstrated that SDS is only beneficial in the presence of egg yolk, suggesting that it functions by altering the tertiary structure of the egg yolk lipoproteins (Pursel et al., 1978). SDS improves the post-thaw survival of spermatozoa by acting as a surfactant to stabilize cell membranes, particularly acrosomal membranes and to protect spermatozoa against the toxic effects of glycerol during the freeze-thaw process (Martin et al., 1979; Arriola and Foote, 1987).

Our results pointed out that, there was no significant effect on sperm viability after thawing when SDS concentration was employed at the highest concentrations $(0.1 ; 0.2$ and $0.4 \% \mathrm{~W} / \mathrm{V})$ as results obtained for the three highest concentrations were very similar. As no variation on the egg yolk or glycerol concentration was analogous in all experiments, one can hypothesize that SDS employed concentrations could enhance the solubility of most of the egg yolk particles, making them more accessible to the spermatozoa, thereby improved the boar spermatozoa's ability to freeze. Moreover, glycerol egg yolk extender containing SDS allows most or all of the egg yolk lipids to be solubilized, thereby improving the protective effects of the extender by reducing the number of unbound SDS molecules that may directly solubilize the sperm membrane lipids which could result in various detrimental effects.

To support our hypothesis, it is important to mention that several investigators have found that the incorporation of SDS in sperm diluents containing egg yolk protected spermatozoa of many species against freezing-induced damage. SDS can also act by solubilizing and increasing the dispersion of egg yolk globules within the diluent, enhancing the contact between protective egg yolk materials and sperm cell membrane (Tsutsui et al., 2000).

In the present study, we were unable to determine the source of the beneficial effects, however, we may speculate that when SDS is used in high concentrations in the extender, the free SDS molecules increase and may bind directly to the sperm membrane, increasing the viability/vitality of the semen after thawing.

\section{Conclusion}

Boar sperm quality after thawing improves significantly when SDS is incorporated into a glycerolegg yolk extender in a concentration between 0.1 to $0.2 \% \mathrm{~W} / \mathrm{V}$ and the best results of viability are obtained for the SDS concentration of $0.2 \% \mathrm{~W} / \mathrm{V}$. Additionally, it is possible to store this extender by freezing it, without decreasing its cryoprotective effect.

\section{Acknowledgment}

This project was financed in $85 \%$ by FEDER and in $15 \%$ with regional funds through the "Programa Operacional Açores 2020 (Operational Program Azores 2020), in scope of the project Cryotec-ACORES-010145-FEDER-000092". Thanks are due to Mr. Jorge Armas and Mr. Mario Coelho for the precious help with boar's semen collecting.

\section{Authors' Contributions}

All authors equally contributed in this study.

\section{Conflict of Interest Declaration}

The authors declare for all legal purposes the absence of any conflict of interest related to this research.

\section{References}

Ali, H. B., Guerin, J. F., Pinatel, M. C., Mathieu, C., Boulieu, D., \& Tritar, B. (1994). Relationship between semen characteristics, a-glucosidase and the capacity of spermatozoa to bind to the human zona pellucida. International Journal of Andrology, 17(3), 121-126. 
Arriola, J., \& Foote, R. H. (1987). Glycerolation and thawing effects on bull spermatozoa frozen in detergent-treated egg yolk and whole egg extenders. Journal of Dairy Science, 70(8), 1664-1670.

Barth, A. D., \& Oko, R. J. (1989). Abnormal morphology of bovine spermatozoa. Iowa State University Press.

Buhr, M. M., \& Pettitt, M. J. (1995). Frozen-thawed boar sperm: isolation of membranes and fluidity measurement. Reproduction in Domestic Animals, 31(1), 147-152.

Cerolini, S., Maldjian, A., Surai, P., \& Noble, R. (2000). Viability, susceptibility to peroxidation and fatty acid composition of boar semen during liquid storage. Animal Reproduction Science, 58(1-2), 99-111.

Cooper, T. G., Yeung, C. H., Nashan, D., Jockenhövel, F., \& Nieschlag, E. (1990). Improvement in the assessment of human epididymal function by the use of inhibitors in the assay of $\alpha$-glucosidase in seminal plasma. International Journal of Andrology, 13(4), 297-305.

Crowe, J. H., Crowe, L. M., Carpenter, J. F., Rudolph, A. S., Wistrom, C. A., Spargo, B. J., \& Anchordoguy, T. J. (1988). Interactions of sugars with membranes. Biochimica et Biophysica Acta (BBA)-Reviews on Biomembranes, 947(2), 367-384.

De Leeuw, F. E., Colenbrander, B., \& Verkleij, A. J. (1991). The role membrane damage plays in cold shock and freezing injury. Reproduction in Domestic Animals, 1, 95-104.

Di Santo, M., Tarozzi, N., Nadalini, M., \& Borini, A. (2011). Human sperm cryopreservation: update on techniques, effect on DNA integrity and implications for ART. Advances in Urology, 2012.

El-Badry, D. A., Anwer, A. M., \& Rawash, Z. M. (2014). Effect of different concentrations of sodium dodecyl sulfate, egg yolk and glycerol on the freezability and DNA integrity of Arabian stallion spermatozoa. Assiut Vet. Med. J, 60, 29-35.

Eriksson, B. M., \& Rodriguez-Martinez, H. (2000). Effect of freezing and thawing rates on the postthaw viability of boar spermatozoa frozen in FlatPacks and Maxi-straws. Animal Reproduction Science, 63(3-4), 205-220.

Esmaeili, V., Shahverdi, A. H., Moghadasian, M. H., \& Alizadeh, A. R. (2015). Dietary fatty acids affect semen quality: a review. Andrology, 3(3), 450-461.

Gaczarzewicz, D., Udala, J., Piasecka, M., Blaszczyk, B., \& Stankiewicz, T. (2016). Bacterial contamination of boar semen and its relationship to sperm quality preserved in commercial extender containing gentamicin sulfate. Polish Journal of Veterinary Sciences, 19(3).

Holt, W. V. (2000). Fundamental aspects of sperm cryobiology: the importance of species and individual differences. Theriogenology, 53(1), 47-58.
Martin, J. C., Klug, E., \& Günzel, A. R. (1979). Centrifugation of stallion semen and its storage in large volume straws. Journal of Reproduction and Fertility. Supplement, (27), 47-51.

Morris, G. J., \& Clarke, A. (1981). Effects of low temperatures on biological membranes.

Paquin, R., Chapdelaine, P., Dubé, J. Y., \& Tremblay, R. R. (1984). Similar biochemical properties of human seminal plasma and epididymal $\alpha$-1, 4-glucosidase. Journal of Andrology, 5(4), 277-282.

Polge, C., Smith, A. U., \& Parkes, A. S. (1949). Revival of spermatozoa after vitrification and dehydration at low temperatures. Nature, 164(4172), 666-666.

Pursel, V. G., Schulman, L. L., \& Johnson, L. A. (1978). Effect of Orvus ES Paste on acrosome morphology, motility and fertilizing capacity of frozen-thawed boar sperm. Journal of Animal Science, 47(1), 198-202.

Roca, J., Hernández, M., Carvajal, G., Vázquez, J. M., \& Martinez, E. A. (2006). Factors influencing boar sperm cryosurvival. Journal of Animal Science, 84(10), 2692-2699.

Rodriguez-Martinez, H., \& Wallgren, M. (2011). Advances in boar semen cryopreservation. Veterinary Medicine International, 2011.

Rusu, A. V., Miclea, V., \& Zăhan, M. (2009). A new model of boar semen evaluation and the impact of cryogenic factor on spermatic cells. Scientific Papers Animal Science and Biotechnologies, 42(1), 85-91.

Silva, C. G., Cunha, E. R., Blume, G. R., Malaquias, J. V., Báo, S. N., \& Martins, C. F. (2015). Cryopreservation of boar sperm comparing different cryoprotectants associated in media based on powdered coconut water, lactose and trehalose. Cryobiology, 70(2), 90-94.

Tsutsui, T., Hase, M., Hori, T., Ito, T., \& Kawakami, E. (2000). Effects of orvus ES paste on canine spermatozoal longevity after freezing and thawing. Journal of Veterinary Medical Science, 62(5), 533-535.

Vilagran Martí, I. (2016). Freezability markers for boar sperm: New Proteomic Approaches.

Westendorf, P., Richter, L., \& Treu, H. (1975). Deep freezing of boar sperma. Laboratory and insemination results using the Hülsenberger paillete method. DTW. Deutsche Tierarztliche Wochenschrift, 82(7), 261.

White, I. G. (1993). Lipids and calcium uptake of sperm in relation to cold shock and preservation: A review. Reproduction, Fertility and Development, 5(6), 639-658.

Yeung, C. H., Cooper, T. G., \& Senge, T. (1990). Histochemical localization and quantification of $\alpha$ glucosidase in the epididymis of men and laboratory animals. Biology of Reproduction, 42(4), 669-676. 